International Journal of Pure and Applied Mathematics

Volume 107 No. $2 \quad 2016,357-369$

ISSN: 1311-8080 (printed version); ISSN: 1314-3395 (on-line version)

url: http://www.ijpam.eu

doi: 10.12732/ijpam.v107i2.6

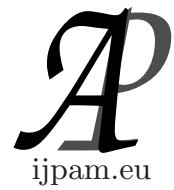

\title{
SEVERAL FIXED POINT THEOREMS FOR EXPANSIVE MAPPINGS IN MULTIPLICATIVE METRIC SPACES
}

\author{
Poonam Nagpal ${ }^{1}$, Shin Min Kang ${ }^{2}$, Sudhir Kumar Garg $^{3}$, Sanjay Kumar ${ }^{4}$ \\ 1,3,4 Department of Mathematics \\ Deenbandhu Chhotu Ram University of Science and Technology \\ Murthal, Sonepat, 131039, Haryana, INDIA \\ ${ }^{2}$ Department of Mathematics and RINS \\ Gyeongsang National University \\ Jinju, 52828, KOREA
}

\begin{abstract}
In this paper, we prove common fixed point results for compatible mappings and its variants satisfying expansive mappings in multiplicative metric spaces. We also give examples in support of our results.
\end{abstract}

AMS Subject Classification: 47H10, 54H 25

Key Words: multiplicative metric space, compatible mapping, compatible mapping of types, expansive mapping

\section{Introduction and Preliminaries}

It is well know that the set of positive real numbers $\mathbb{R}_{+}$is not complete according to the usual metric. To overcome this problem, in 2008, Bashirov et al. [2] introduced the concept of multiplicative metric spaces as follows:

Definition 1.1. Let $X$ be a nonempty set. A multiplicative metric is a mapping $d: X \times X \rightarrow \mathbb{R}_{+}$satisfying the following conditions:

(i) $d(x, y) \geq 1$ for all $x, y \in X$ and $d(x, y)=1$ if and only if $x=y$;

(ii) $d(x, y)=d(y, x)$ for all $x, y \in X$;

Received: January 19, 2016

Published: April 4, 2016

${ }^{\S}$ Correspondence author (c) 2016 Academic Publications, Ltd.

url: www.acadpubl.eu 
(iii) $d(x, y) \leq d(x, z) \cdot d(z, y)$ for all $x, y, z \in X$ (multiplicative triangle inequality).

Then the mapping $d$ together with $X$, that is, $(X, d)$ is a multiplicative metric space.

Example 1.2. ([6]) Let $\mathbb{R}_{+}^{n}$ be the collection of all $n$-tuples of positive real numbers. Let $d^{*}: \mathbb{R}_{+}^{n} \times \mathbb{R}_{+}^{n} \rightarrow \mathbb{R}$ be defined as follows:

$$
d^{*}(x, y)=\left|\frac{x_{1}}{y_{1}}\right|^{*} \cdot\left|\frac{x_{2}}{y_{2}}\right|^{*} \ldots\left|\frac{x_{n}}{y_{n}}\right|^{*}
$$

where $x=\left(x_{1}, \ldots, x_{n}\right), y=\left(y_{1}, \ldots, y_{n}\right) \in \mathbb{R}_{+}^{n}$ and $|\cdot|^{*}: \mathbb{R}_{+} \rightarrow \mathbb{R}_{+}$is defined by

$$
|a|^{*}= \begin{cases}a & \text { if } a \geq 1 \\ \frac{1}{a} & \text { if } a<1\end{cases}
$$

Then it is obvious that all conditions of a multiplicative metric are satisfied. Therefore $\left(\mathbb{R}_{+}^{n}, d^{*}\right)$ is a multiplicative metric space.

Example 1.3. ([8]) Let $d: \mathbb{R} \times \mathbb{R} \rightarrow[1, \infty)$ be defined as $d(x, y)=a^{|x-y|}$, where $x, y \in \mathbb{R}$ and $a>1$. Then $d$ is a multiplicative metric and $(\mathbb{R}, d)$ is a multiplicative metric space. We may call it usual multiplicative metric spaces.

Remark 1.4. We note that the Example 1.2 is valid for positive real numbers and Example 1.3 is valid for all real numbers.

Example 1.5. ([8]) Let $(X, d)$ be a metric space. Define a mapping $d_{a}$ on $X$ by

$$
d_{a}(x, y)=a^{d(x, y)}= \begin{cases}1 & \text { if } x=y \\ a & \text { if } x \neq y\end{cases}
$$

where $x, y \in X$ and $a>1$. Then $d_{a}$ is a multiplicative metric and $\left(X, d_{a}\right)$ is known as the discrete multiplicative metric space.

Example 1.6. ([1]) Let $X=C^{*}[a, b]$ be the collection of all real-valued multiplicative continuous functions on $[a, b] \subset \mathbb{R}_{+}$. Then $(X, d)$ is a multiplicative metric space with $d$ defined by $d(f, g)=\sup _{x \in[a, b]}\left|\frac{f(x)}{g(x)}\right|$ for arbitrary $f, g \in X$.

Remark 1.7. ([8]) We note that multiplicative metrics and metric spaces are independent.

Indeed, the mapping $d^{*}$ defined in Example 1.2 is multiplicative metric but 
not metric as it does not satisfy triangular inequality. Consider

$$
d^{*}\left(\frac{1}{3}, \frac{1}{2}\right)+d^{*}\left(\frac{1}{2}, 3\right)=\frac{3}{2}+6=7.5<9=d^{*}\left(\frac{1}{3}, 3\right) .
$$

On the other hand the usual metric on $\mathbb{R}$ is not multiplicative metric as it doesnt satisfy multiplicative triangular inequality, since

$$
d(2,3) \cdot d(3,6)=3<4=d(2,6)
$$

One can refer to $[3,6]$ for detailed multiplicative metric topology.

Definition 1.8. Let $(X, d)$ be a multiplicative metric space. Then a sequence $\left\{x_{n}\right\}$ in $X$ said to be

(1) a multiplicative convergent to $x$ if for every multiplicative open ball $B_{\epsilon}(x)=\{y \mid d(x, y)<\epsilon\}, \epsilon>1$, there exists a natural number $N$ such that $n \geq N$, then $x_{n} \in B_{\epsilon}(x)$, that is, $d\left(x_{n}, x\right) \rightarrow 1$ as $n \rightarrow \infty$.

(2) a multiplicative Cauchy sequence if for all $\epsilon>1$, there exists $N \in \mathbb{N}$ such that $d\left(x_{n}, x_{m}\right)<\epsilon$ for all $m, n>N$, that is, $d\left(x_{n}, x_{m}\right) \rightarrow 1$ as $n, m \rightarrow \infty$.

(3) We call a multiplicative metric space complete if every multiplicative Cauchy sequence in it is multiplicative convergent to $x \in X$.

Remark 1.9. The set of positive real numbers $\mathbb{R}_{+}$is not complete according to the usual metric. Let $X=\mathbb{R}_{+}$and the sequence $\left\{x_{n}\right\}=\left\{\frac{1}{n}\right\}$. It is obvious $\left\{x_{n}\right\}$ is a Cauchy sequence in $X$ with respect to usual metric and $X$ is not a complete metric space, since $0 \notin \mathbb{R}_{+}$. In case of a multiplicative metric space, we take a sequence $\left\{x_{n}\right\}=\left\{a^{\frac{1}{n}}\right\}$, where $a>1$. Then $\left\{x_{n}\right\}$ is a multiplicative Cauchy sequence since for $n \geq m$,

$$
\begin{aligned}
d\left(x_{n}, x_{m}\right) & =\left|\frac{x_{n}}{x_{m}}\right|=\left|\frac{a^{\frac{1}{n}}}{a^{\frac{1}{m}}}\right|=\left|a^{\frac{1}{n}-\frac{1}{m}}\right| \\
& \leq a^{\frac{1}{m}-\frac{1}{n}}<a^{\frac{1}{m}}<\epsilon \text { if } m>\frac{\log a}{\log \epsilon}
\end{aligned}
$$

where $|a|=\left\{\begin{array}{ll}a & \text { if } a \geq 1, \\ \frac{1}{a} & \text { if } a<1 .\end{array}\right.$ Also, $\left\{x_{n}\right\} \rightarrow 1$ as $n \rightarrow \infty$ and $1 \in \mathbb{R}_{+}$. Hence $(X, d)$ is a complete multiplicative metric space.

In 2012, Özavsar and Çevikel [6] gave the concept of multiplicative contraction mappings and proved some fixed point theorems of such mappings in a multiplicative metric space. 
Definition 1.10. Let $f$ be a mapping of a multiplicative metric space $(X, d)$ into itself. Then $f$ is said to be a multiplicative contraction if there exists a real constant $\lambda \in[0,1)$ such that

$$
d(f x, f y) \leq d^{\lambda}(x, y) \text { for all } x, y \in X .
$$

Also they proved the Banach Contraction Principle in the setting of multiplicative metric spaces as follows:

Theorem 1.11. Let $f$ be a multiplicative contraction mapping of a complete multiplicative metric space $(X, d)$ into itself. Then $f$ has a unique fixed point.

In 1984, Wang et al. [9] and in 1993, Rhoades [7] proved some fixed point theorems for expansion mappings, which corresponds to some contractive mappings in metric spaces.

Now we define expansive mappings in the setting of multiplicative metric spaces as follows:

Definition 1.12. Let $f$ be a mapping of a multiplicative metric space $(X, d)$ into itself. Then $f$ is said to be an expansive mapping if there exists a constant $\alpha>1$ such that for all $x, y \in X$, we have

$$
d(f x, f y) \geq d^{\alpha}(x, y) .
$$

Example 1.13. Let $X=\mathbb{R}_{+}$with usual multiplicative metric $d$. Define $f: X \rightarrow X$ by

$$
f x= \begin{cases}\frac{1}{x^{4}} & \text { if } x \neq 1 \\ 1 & \text { if } x=1\end{cases}
$$

Then clearly,

$$
d(f x, f y) \geq d^{\alpha}(x, y)
$$

holds for $1<\alpha \leq 4$, which implies that $f$ is an expansive mapping.

Recently Kang et al. [5] introduced the notion of compatible mappings and its variants as follows:

Definition 1.14. Let $f$ and $g$ be two mappings of a multiplicative metric space $(X, d)$ into itself. Then $f$ and $g$ are called

(1) compatible if

$$
\lim _{n \rightarrow \infty} d\left(f g x_{n}, g f x_{n}\right)=1,
$$

whenever $\left\{x_{n}\right\}$ is a sequence in $X$ such that $\lim _{n \rightarrow \infty} f x_{n}=\lim _{n \rightarrow \infty} g x_{n}=t$ for some $t \in X$. 
(2) compatible of type $(A)$ if

$$
\lim _{n \rightarrow \infty} d\left(f g x_{n}, g g x_{n}\right)=1 \text { and } \lim _{n \rightarrow \infty} d\left(g f x_{n}, f f x_{n}\right)=1
$$

whenever $\left\{x_{n}\right\}$ is a sequence in $X$ such that $\lim _{n \rightarrow \infty} f x_{n}=\lim _{n \rightarrow \infty} g x_{n}=t$ for some $t \in X$.

(3) compatible of type $(B)$ if

$$
\lim _{n \rightarrow \infty} d\left(f g x_{n}, g g x_{n}\right) \leq\left[\lim _{n \rightarrow \infty} d\left(f g x_{n}, f t\right) \cdot \lim _{n \rightarrow \infty} d\left(f t, f f x_{n}\right)\right]^{1 / 2}
$$

and

$$
\lim _{n \rightarrow \infty} d\left(g f x_{n}, f f x_{n}\right) \leq\left[\lim _{n \rightarrow \infty} d\left(g f x_{n}, g t\right) \cdot \lim _{n \rightarrow \infty} d\left(g t, g g x_{n}\right)\right]^{1 / 2},
$$

whenever $\left\{x_{n}\right\}$ is a sequence in $X$ such that $\lim _{n \rightarrow \infty} f x_{n}=\lim _{n \rightarrow \infty} g x_{n}=t$ for some $t \in X$.

(4) compatible of type $(C)$ if

$$
\begin{aligned}
& \lim _{n \rightarrow \infty} d\left(f g x_{n}, g g x_{n}\right) \\
& \leq\left[\lim _{n \rightarrow \infty} d\left(f g x_{n}, f t\right) \cdot \lim _{n \rightarrow \infty} d\left(f t, f f x_{n}\right) \cdot \lim _{n \rightarrow \infty} d\left(f t, g g x_{n}\right)\right]^{1 / 3}
\end{aligned}
$$

and

$$
\begin{aligned}
& \lim _{n \rightarrow \infty} d\left(g f x_{n}, f f x_{n}\right) \\
& \leq\left[\lim _{n \rightarrow \infty} d\left(g f x_{n}, g t\right) \cdot \lim _{n \rightarrow \infty} d\left(g t, g g x_{n}\right) \cdot \lim _{n \rightarrow \infty} d\left(g t, f f x_{n}\right)\right]^{1 / 3},
\end{aligned}
$$

whenever $\left\{x_{n}\right\}$ is a sequence in $X$ such that $\lim _{n \rightarrow \infty} f x_{n}=\lim _{n \rightarrow \infty} g x_{n}=t$ for some $t \in X$

(5) compatible of type $(P)$ if

$$
\lim _{n \rightarrow \infty} d\left(f f x_{n}, g g x_{n}\right)=1
$$

whenever $\left\{x_{n}\right\}$ is a sequence in $X$ such that $\lim _{n \rightarrow \infty} f x_{n}=\lim _{n \rightarrow \infty} g x_{n}=t$ for some $t \in X$.

Now we state some results which are useful for our main results.

Proposition 1.15. Let $f$ and $g$ be compatible mappings of a multiplicative metric space $(X, d)$ into itself. Suppose that $\lim _{n \rightarrow \infty} f x_{n}=\lim _{n \rightarrow \infty} g x_{n}=t$ for some $t \in X$. Then $\lim _{n \rightarrow \infty} g f x_{n}=f t$ if $f$ is continuous at $t$. 
Proposition 1.16. Let $f$ and $g$ be compatible mappings of type $(A)$ of a multiplicative metric space $(X, d)$ into itself. If one of $f$ and $g$ is continuous, then $f$ and $g$ are compatible.

Recently, Kang et al. [4] also introduced the notion of compatible mappings of type $(R)$, of type $(K)$ and of type $(E)$ as follows:

Definition 1.17. Let $f$ and $g$ be two mappings of a multiplicative metric space $(X, d)$ into itself. Then $f$ and $g$ are called

(1) compatible of type $(R)$ if

$$
\lim _{n \rightarrow \infty} d\left(f g x_{n}, g f x_{n}\right)=1 \text { and } \lim _{n \rightarrow \infty} d\left(f f x_{n}, g g x_{n}\right)=1,
$$

whenever $\left\{x_{n}\right\}$ is a sequence in $X$ such that $\lim _{n \rightarrow \infty} f x_{n}=\lim _{n \rightarrow \infty} g x_{n}=t$ for some $t \in X$.

(2) compatible of type $(K)$ if

$$
\lim _{n \rightarrow \infty} d\left(f f x_{n}, g t\right)=1 \text { and } \lim _{n \rightarrow \infty} d\left(g g x_{n}, f t\right)=1,
$$

whenever $\left\{x_{n}\right\}$ is a sequence in $X$ such that $\lim _{n \rightarrow \infty} f x_{n}=\lim _{n \rightarrow \infty} g x_{n}=t$ for some $t \in X$.

(3) compatible of type $(E)$ if

$$
\lim _{n \rightarrow \infty} f f x_{n}=\lim _{n \rightarrow \infty} f g x_{n}=g t \quad \text { and } \quad \lim _{n \rightarrow \infty} g g x_{n}=\lim _{n \rightarrow \infty} g f x_{n}=f t,
$$

whenever $\left\{x_{n}\right\}$ is a sequence in $X$ such that $\lim _{n \rightarrow \infty} f x_{n}=\lim _{n \rightarrow \infty} g x_{n}=t$ for some $t \in X$.

Remark 1.18. Obviously, compatible mappings of type $(R)$ are also compatible mappings and compatible mappings of type $(P)$.

\section{Main Results}

In 1993, Rhoades [7] proved the following fixed point theorem for expansive mappings in metric spaces as follows:

Theorem 2.1. Let $f$ and $g$ be compatible mappings of a complete metric space into itself satisfying the condition

$$
d(f x, f y) \geq q d(g x, g y)
$$

for all $x, y \in X$, where $q>1$.

Assume that $g(X) \subset f(X)$ and $f$ is continuous. Then $f$ and $g$ have a unique common fixed point. 
Now we are proving Theorem 2.1 in the setting of multiplicative metric spaces in the following way:

Theorem 2.2. Let $f$ and $g$ be compatible mappings of a complete multiplicative metric space into itself satisfying the condition

$$
d(f x, f y) \geq d^{q}(g x, g y)
$$

for all $x, y \in X$, where $q>1$.

Assume that $g(X) \subset f(X)$ and $f$ is continuous. Then $f$ and $g$ have a unique common fixed point.

Proof. Let $x_{0} \in X$. Since $g(X) \subset f(X)$, there exists $x_{1} \in X$ such that $f x_{1}=g x_{0}$. In general, there exists $x_{n+1} \in X$ such that

$$
y_{n}=f x_{n+1}=g x_{n} .
$$

From (2.1), consider

$$
\begin{aligned}
d\left(y_{n}, y_{n+1}\right) & =d\left(g x_{n}, g x_{n+1}\right) \leq d^{1 / q}\left(f x_{n}, f x_{n+1}\right) \\
& \leq d^{1 / q}\left(g x_{n-1}, g x_{n}\right) \leq d^{1 / q^{2}}\left(f x_{n-1}, f x_{n}\right) \\
& =d^{1 / q^{2}}\left(y_{n-2}, y_{n-1}\right) .
\end{aligned}
$$

In general, we have

$$
d\left(y_{n}, y_{n+1}\right) \leq d^{k^{n}}\left(y_{0}, y_{1}\right)
$$

where $k=1 / q<1$.

Now for $m, n \in \mathbb{N}$ with $n<m$, consider

$$
\begin{aligned}
d\left(y_{n}, y_{m}\right) & \leq d\left(y_{n}, y_{n+1}\right) \cdot d\left(y_{n+1}, y_{n+2}\right) \cdots d\left(y_{m-1}, y_{m}\right) \\
& \leq d^{k^{n}}\left(y_{0}, y_{1}\right) \cdot d^{k^{n+1}}\left(y_{0}, y_{1}\right) \cdots d^{k^{m-1}}\left(y_{0}, y_{1}\right) \\
& \leq d^{\frac{k^{n}}{1-k}}\left(y_{0}, y_{1}\right) \\
& \rightarrow 1
\end{aligned}
$$

as $n \rightarrow \infty$. It follows that the sequence $\left\{y_{n}\right\}$ is a multiplicative Cauchy sequence. Since $(X, d)$ is complete, we have

$$
\lim _{n \rightarrow \infty} f x_{n}=\lim _{n \rightarrow \infty} g x_{n}=z .
$$

Since $f$ and $g$ are compatible and $f$ is continuous, by Proposition 1.15,

$$
\lim _{n \rightarrow \infty} f f x_{n}=\lim _{n \rightarrow \infty} f g x_{n}=\lim _{n \rightarrow \infty} g f x_{n}=f z .
$$


Consider

$$
d\left(g f x_{n}, g z\right) \leq d^{1 / q}\left(f f x_{n}, f z\right) .
$$

Letting $n \rightarrow \infty$, we get

$$
\lim _{n \rightarrow \infty} g f x_{n}=g z=f z .
$$

Now we show that $z$ is fixed point of $f$ and $g$. Again considering

$$
d\left(g z, g x_{n}\right) \leq d^{1 / q}\left(f z, f x_{n}\right) .
$$

Letting $n \rightarrow \infty$, we get

$$
d(g z, z) \leq d^{1 / q}(f z, z),
$$

which implies that $f z=z$. Hence $z$ is fixed point of $f$ and $g$.

Uniqueness follows easily from (2.1). This completes the proof.

Example 2.3. Let $X=\mathbb{R}_{+}$with the usual multiplicative metric $d$. Define $f$ and $g: X \rightarrow X$ by

$$
f x=1 / x^{4} \text { and } g x=1 / x^{2}
$$

for all $x \in X$. Then clearly $f$ and $g$ are compatible mappings as $\lim _{n \rightarrow \infty} d\left(f g x_{n}\right.$, $\left.g f x_{n}\right)=1$. Also for $1<q \leq 2$, the condition (2.1) holds. Further $g(X) \subset f(X)$ and $f$ is continuous. Then from Theorem 2.2, $f$ and $g$ have unique common fixed point 1.

Next we prove a common fixed point theorem for compatible mappings of type $(A)$ as follows:

Theorem 2.4. Let $f$ and $g$ be compatible mappings of type $(A)$ of a complete multiplicative metric space into itself satisfying the condition (2.1).

Assume that $g(X) \subset f(X)$ and $f$ is continuous. Then $f$ and $g$ have a unique common fixed point.

Proof. Since $f$ is continuous and $f$ and $g$ are compatible of type $(A)$, therefore, by Proposition 1.16, the pair $f, g$ is compatible. The conclusion follows easily from Theorem 2.2 .

Example 2.5. Let $X=\mathbb{R}_{+}$with the usual multiplicative metric $d$. Define $f$ and $g: X \rightarrow X$ by

$$
f x=x^{2} \quad \text { and } \quad g x=\sqrt{x}
$$

for all $x \in X$. Let $\left\{x_{n}\right\}$ be a sequence in $X$ such that $\lim _{n \rightarrow \infty} f x_{n}=\lim _{n \rightarrow \infty} g x_{n}=t$ for some $t \in X$, which implies that $\lim _{n \rightarrow \infty} x_{n}^{2}=\lim _{n \rightarrow \infty} \sqrt{x_{n}}=t$ for some $t \in X$. 
Now we have

$$
\lim _{n \rightarrow \infty} f g x_{n}=\lim _{n \rightarrow \infty} x_{n}=\sqrt{t}=\lim _{n \rightarrow \infty} x_{n}^{1 / 4}=\lim _{n \rightarrow \infty} g g x_{n}
$$

and

$$
\lim _{n \rightarrow \infty} g f x_{n}=\lim _{n \rightarrow \infty} x_{n}=t^{2}=\lim _{n \rightarrow \infty} x_{n}^{4}=\lim _{n \rightarrow \infty} f f x_{n},
$$

which implies that $f$ and $g$ are compatible mappings of type $(A)$.

Also for $1<q \leq 3 / 2$, the condition (2.1) holds. Further $g(X) \subset f(X)$ and $f$ is continuous. Then from Theorem 2.4, $f$ and $g$ have unique common fixed point 1 .

Next we prove a common fixed point theorem for compatible mappings of type $(B)$ as follows:

Theorem 2.6. Let $f$ and $g$ be compatible mappings of type $(B)$ of a complete multiplicative metric space into itself satisfying the condition (2.1).

Assume that $g(X) \subset f(X)$ and $f$ is continuous. Then $f$ and $g$ have a unique common fixed point.

Proof. From the proof of Theorem 2.2, $\left\{g x_{n}\right\}$ is a multiplicative Cauchy sequence. Since $(X, d)$ is complete, there exists a point $z \in X$ such that

$$
\lim _{n \rightarrow \infty} f x_{n}=\lim _{n \rightarrow \infty} g x_{n}=z .
$$

Since $f$ is continuous, we have

$$
\lim _{n \rightarrow \infty} f f x_{n}=\lim _{n \rightarrow \infty} f g x_{n}=f z .
$$

Since $f$ and $g$ are compatible of type $(B)$, so

$$
\lim _{n \rightarrow \infty} d\left(f g x_{n}, g g x_{n}\right) \leq\left[\lim _{n \rightarrow \infty} d\left(f g x_{n}, f z\right) \cdot \lim _{n \rightarrow \infty} d\left(f z, f f x_{n}\right)\right]^{1 / 2} .
$$

Letting $n \rightarrow \infty$, we have

$$
\lim _{n \rightarrow \infty} d\left(f z, g g x_{n}\right) \leq[d(f z, f z) \cdot d(f z, f z)]^{1 / 2},
$$

which implies that $\lim _{n \rightarrow \infty} g g x_{n}=f z$.

Consider

$$
\lim _{n \rightarrow \infty} d\left(g g x_{n}, g f x_{n}\right)=\lim _{n \rightarrow \infty} d\left(g g x_{n}, f f x_{n}\right)=d^{1 / q}(f z, f z),
$$


which implies that

$$
\lim _{n \rightarrow \infty} g g x_{n}=\lim _{n \rightarrow \infty} g f x_{n}=f z .
$$

Again consider

$$
d\left(g f x_{n}, g z\right) \leq d^{1 / q}\left(f f x_{n}, f z\right) .
$$

Letting $n \rightarrow \infty$, we have $f z=g z$.

The rest of the proof follows easily from Theorem 2.2.

Now we prove a common fixed point theorem for compatible mappings of type $(C)$ as follows:

Theorem 2.7. Let $f$ and $g$ be compatible mappings of type $(C)$ of a complete multiplicative metric space into itself satisfying the condition (2.1).

Assume that $g(X) \subset f(X)$ and $f$ is continuous. Then $f$ and $g$ have a unique common fixed point.

Proof. From the proof of Theorem 2.2, $\left\{g x_{n}\right\}$ is a multiplicative Cauchy sequence. Since $(X, d)$ is complete, there exists a point $z \in X$ such that

$$
\lim _{n \rightarrow \infty} f x_{n}=\lim _{n \rightarrow \infty} g x_{n}=z .
$$

Since $f$ is continuous, we have

$$
\lim _{n \rightarrow \infty} f f x_{n}=\lim _{n \rightarrow \infty} f g x_{n}=f z .
$$

Since $f$ and $g$ are compatible of type $(C)$, so

$$
\begin{aligned}
& \lim _{n \rightarrow \infty} d\left(f g x_{n}, g g x_{n}\right) \\
& \leq\left[\lim _{n \rightarrow \infty} d\left(f g x_{n}, f z\right) \cdot \lim _{n \rightarrow \infty} d\left(f z, f f x_{n}\right) \cdot \lim _{n \rightarrow \infty} d\left(f z, g g x_{n}\right)\right]^{1 / 3} .
\end{aligned}
$$

Letting $n \rightarrow \infty$, we have

$$
\lim _{n \rightarrow \infty} d\left(f z, g g x_{n}\right) \leq\left[d(f z, f z) \cdot d(f z, f z) \cdot \lim _{n \rightarrow \infty} d\left(f z, g g x_{n}\right)\right]^{1 / 3},
$$

which implies that $\lim _{n \rightarrow \infty} g g x_{n}=f z$.

Consider

$$
\lim _{n \rightarrow \infty} d\left(g g x_{n}, g f x_{n}\right) \leq \lim _{n \rightarrow \infty} d^{1 / q}\left(f g x_{n}, f f x_{n}\right)=d^{1 / q}(f z, f z),
$$

which implies that

$$
\lim _{n \rightarrow \infty} g g x_{n}=\lim _{n \rightarrow \infty} g f x_{n}=f z .
$$


Now consider

$$
d\left(g f x_{n}, g z\right) \leq d^{1 / q}\left(f f x_{n}, f z\right) .
$$

Letting $n \rightarrow \infty$, we have $f z=g z$.

The rest of the proof follows easily from Theorem 2.2.

Now we prove a common fixed point theorem for compatible mappings of type $(P)$ as follows:

Theorem 2.8. Let $f$ and $g$ be compatible mappings of type $(P)$ of a complete multiplicative metric space into itself satisfying the condition (2.1).

Assume that $g(X) \subset f(X)$ and $f$ is continuous. Then $f$ and $g$ have a unique common fixed point.

Proof. From the proof of Theorem 2.2, $\left\{g x_{n}\right\}$ is a multiplicative Cauchy sequence. Since $(X, d)$ is complete, there exists $z \in X$ such that

$$
\lim _{n \rightarrow \infty} f x_{n}=\lim _{n \rightarrow \infty} g x_{n}=z .
$$

Since $f$ and $g$ are compatible of type $(P)$ and $f$ is continuous, we have

$$
\lim _{n \rightarrow \infty} f f x_{n}=\lim _{n \rightarrow \infty} g g x_{n}=\lim _{n \rightarrow \infty} f g x_{n}=f z .
$$

Consider

$$
\lim _{n \rightarrow \infty} d\left(g g x_{n}, g z\right) \leq \lim _{n \rightarrow \infty} d^{1 / q}\left(f g x_{n}, f z\right)
$$

which implies that

$$
\lim _{n \rightarrow \infty} g g x_{n}=g z=f z .
$$

The rest of the proof follows easily from Theorem 2.2 .

Now we prove a common fixed point theorem for compatible mappings of type $(R)$ as follows:

Theorem 2.9. Let $f$ and $g$ be compatible mappings of type $(R)$ of a complete multiplicative metric space into itself satisfying the condition (2.1).

Assume that $g(X) \subset f(X)$ and $f$ is continuous. Then $f$ and $g$ have a unique common fixed point.

Proof. It follows from Remark 1.18 and Theorem 2.2.

Now we prove a common fixed point theorem for compatible mappings of type $(K)$ as follows: 
Theorem 2.10. Let $f$ and $g$ be compatible mappings of type $(K)$ of a complete multiplicative metric space into itself satisfying the condition (2.1).

Assume that $g(X) \subset f(X)$ and $f$ is continuous. Then $f$ and $g$ have a unique common fixed point.

Proof. From the proof of Theorem 2.2, $\left\{g x_{n}\right\}$ is a multiplicative Cauchy sequence. Since $(X, d)$ is complete, there exists $z \in X$ such that

$$
\lim _{n \rightarrow \infty} f x_{n}=\lim _{n \rightarrow \infty} g x_{n}=z .
$$

Since $f$ and $g$ are compatible of type $(K)$ and $f$ is continuous, we have

$$
\lim _{n \rightarrow \infty} f f x_{n}=g z=f z .
$$

The rest of the proof follows easily from Theorem 2.2.

Finally, we prove a common fixed point theorem for compatible mappings of type $(E)$ as follows:

Theorem 2.11. Let $f$ and $g$ be compatible mappings of type $(E)$ of a complete multiplicative metric space into itself satisfying the condition (2.1).

Assume that $g(X) \subset f(X)$ and $f$ is continuous. Then $f$ and $g$ have a unique common fixed point.

Proof. From the proof of Theorem 2.2, $\left\{g x_{n}\right\}$ is Cauchy sequence. Since $(X, d)$ is complete, there exists $z \in X$ such that

$$
\lim _{n \rightarrow \infty} f x_{n}=\lim _{n \rightarrow \infty} g x_{n}=z
$$

Since $f$ and $g$ are compatible of type $(E)$ and $f$ is continuous, we have

$$
\lim _{n \rightarrow \infty} f f x_{n}=\lim _{n \rightarrow \infty} f g x_{n}=g z=f z .
$$

The rest of the proof follows easily from Theorem 2.2.

\section{References}

[1] M. Abbas, B. Ali, Y.I. Suleiman, Common fixed points of locally contractive mappings in multiplicative metric spaces with application, Int. J. Math. Math. Sci., 2015 (2015), Article ID 218683, 7 pages. doi:10.1155/2015/218683.

[2] A.E. Bashirov, E.M. Kurplnara, A. Ozyapici, Multiplicative calculus and its applications, J. Math. Anal. Appl., 337 (2008), 36-48. doi: 10.1016/j.jmaa.2007.03.081 
[3] X. He, M. Song, D. Chen, Common fixed points for weak commutative mappings on a multiplicative metric space, Fixed Point Theory Appl., 48 (2014), 9 pages. doi: $10.1186 / 1687-1812-2014-48$

[4] S.M. Kang, P. Kumar, S. Kumar, Common fixed points for compatible mappings of types in multiplicative metric spaces, Int. J. Math. Anal., 9 (2015), 1755-1767. doi: 10.12988/ijma.2015.53104

[5] S.M. Kang, P. Kumar, S. Kumar, P. Nagpal, S.K. Garg, Common fixed points for compatible mappings and its variants in multiplicative metric spaces, Int. J. Pure Appl. Math., 102 (2015), 383-406. doi: 10.12732/ijpam.v102i2.14

[6] M. Özavsar, A.C. Çevikel, Fixed points of multiplicative contraction mappings on multiplicative metric spaces, arXiv:1205.5131v1 [math.GM], 2012.

[7] B.E. Rhoades, An expansion mapping theorem, Jñānābha, 23 (1993), 151-152.

[8] M. Sarwar, R. Badshah-e, Some unique fixed point theorems in multiplicative metric space, arXiv:1410.3384v2 [math.GM], 2014.

[9] S.Z. Wang, B.Y, Li, Z.M. Gao, K. Iséki, Some fixed point theorems on expansion mappings, Math. Japon., 29 (1984), 631-636. 
